\title{
TWO-ROW NILPOTENT ORBITS OF CYCLIC QUIVERS
}

\author{
ANTHONY HENDERSON
}

\begin{abstract}
We prove that the local intersection cohomology of nilpotent orbit closures of cyclic quivers is trivial when the two orbits involved correspond to partitions with at most two rows. This gives a geometric proof of a result of Graham and Lehrer, which states that standard modules of the affine Hecke algebra of $G L_{d}$ corresponding to nilpotents with at most two Jordan blocks are multiplicity-free.
\end{abstract}

\section{INTRODUCTION}

Let $\Delta_{n}$ be the cyclic quiver with $n$ vertices. The isomorphism classes of nilpotent complex representations of $\Delta_{n}$ are in bijection with certain nilpotent orbits in a suitable variety (see $\S 2$ for the definition). The closure of such a nilpotent orbit is usually singular, and it is an important problem to compute its local intersection cohomology at the points of another given orbit (see $\S 3$ ). If $n=1$, these are the usual nilpotent orbits in $\mathfrak{g l}_{d}$, and the problem was solved by Lusztig in [6].

The main result of this paper (Theorem 3.1 below) is that this local intersection cohomology is trivial when the two orbits involved correspond to partitions with at most two rows (equivalently, the representations of $\Delta_{n}$ involved are either indecomposable or the sum of two indecomposables).

In $\S 3$ we discuss some equivalent formulations of this result. The most noteworthy concerns the complex representation theory of $\widetilde{\mathcal{H}}_{d}$, the affine Hecke algebra of $G L_{d}(\mathbb{C})$ (as defined in [4]). This algebra has geometrically-defined standard modules $M_{s, x, q_{0}}$ indexed by triples $\left(s, x, q_{0}\right)$ where $s \in G L_{d}(\mathbb{C})$ is semisimple, $x \in \mathfrak{g l}_{d}(\mathbb{C})$ is nilpotent, $q_{0} \in \mathbb{C}^{\times}$, and

$$
\operatorname{Ad}(s)(x)=q_{0} x .
$$

By a well-known result of Ginzburg, Theorem 3.1 (for $n \geq 2$ ) is equivalent to the statement that $M_{s, x, q_{0}}$ is multiplicity-free when $q_{0}$ is a primitive $n$-th root of unity, all eigenvalues of $s$ are powers of $q_{0}$, and $x$ has at most two Jordan blocks. (See $\S 3$ for more on the equivalence.) 
This statement was recently proved by Graham and Lehrer in [2]. Actually, they imposed no conditions on $s$ and $q_{0}$, but it is easy to reduce to the above case. Their method is algebraic, making use of the representation theory of the affine Temperley-Lieb algebras, and their results apply over more general ground fields (with induced modules instead of standard modules). The present paper arose from the author's desire to give a purely geometric/combinatorial proof of Theorem 3.1, thus reproving Graham and Lehrer's result (in the complex case).

In $\S 4$ we introduce canonical resolutions of the nilpotent orbit closures, and derive a dimension formula for the orbits, which appears to be new (see Lemma 4.1). We also discuss how to determine the local intersection cohomology, given the Poincaré polynomials of the fibres of these resolutions. Then for the remainder of the paper we restrict attention to the nilpotent orbits with at most two rows. Section 5 contains some preliminary Lemmas, and Section 6 completely describes the closure relations among such orbits. Section 7 presents the proof of Theorem 3.1.

Our notation for partitions and Young diagrams follows 8 . In particular, if $\lambda$ is a partition, its nonzero parts are $\lambda_{1} \geq \lambda_{2} \geq \cdots \geq \lambda_{\ell(\lambda)}$, where $\ell(\lambda)$ is the length; and $\lambda^{\prime}$ denotes the transpose partition.

Acknowledgements. I am very grateful to John Graham and Gus Lehrer for telling me about their result and showing me a draft of [2].

\section{Nilpotent Orbits of Cyclic Quivers}

Fix a positive integer $n$. Let $\Delta_{n}$ be the cyclic quiver of type $\widetilde{A_{n-1}}$, which has vertex set $I=\mathbb{Z} / n \mathbb{Z}$ and an arrow from $i$ to $i-1$ for all $i \in I$. Let $V=\oplus_{i \in I} V_{i}$ be an $I$-graded vector space over $\mathbb{C}$. We write $d_{i}$ for $\operatorname{dim} V_{i}$, d for the dimension vector $\operatorname{dim} V=\left(d_{i}\right)_{i \in I}$, and $d$ for $\operatorname{dim} V=\sum_{i \in I} d_{i}$. Let

$$
E_{V}=\bigoplus_{i \in I} \operatorname{Hom}\left(V_{i}, V_{i-1}\right)
$$

be the space of representations of $\Delta_{n}$ on $V$. Two representations in $E_{V}$ are isomorphic iff they are in the same orbit of $G_{V}=\prod_{i \in I} G L\left(V_{i}\right)$, acting on $E_{V}$ by conjugation.

We say that $x \in E_{V}$, and its $G_{V}$-orbit, are nilpotent if $x$ is nilpotent as an element of $\operatorname{End}(V)$. Let $\mathcal{N}_{V}$ be the subvariety of nilpotent elements of $E_{V}$. The nilpotent $G L(V)$-orbits in $\operatorname{End}(V)$ are in bijection with $\Lambda(d)$, the set of partitions of size $d$ : let $\mathcal{O}_{\lambda}$ denote the orbit corresponding to the partition $\lambda$. The $G_{V}$-orbits in $\mathcal{N}_{V}$ have a similar description, as follows. 
The isomorphism classes of indecomposable nilpotent representations of $\Delta_{n}$ are called segments. There is a segment $[i ; l)$ for each $i \in I$ and $l \in \mathbb{Z}^{+}$; in our convention this is the isoclass of indecomposables of length $l$ whose socle is the simple module corresponding to $i$. The notation is meant to suggest the multiset $\{i, i+1, \cdots, i+l-1\}$, which (regarded as a function $I \rightarrow \mathbb{N}$ ) is precisely the dimension vector of an indecomposable representation in this class. Since any representation is a direct sum of indecomposables, the nilpotent representations are parametrized by multisegments (multisets of segments), which we write with direct sum notation, e.g. $\left[i_{1} ; l_{1}\right) \oplus \cdots \oplus\left[i_{s} ; l_{s}\right)$.

Collecting together all segments $[i ; l)$ with the same $i$, we can think of a multisegment as an $I$-tuple of partitions $\boldsymbol{\lambda}=\left(\lambda^{(i)}\right)_{i \in I}$. Let $\boldsymbol{\Lambda}$ be the set of such $I$-tuples, and for $\boldsymbol{\lambda} \in \boldsymbol{\Lambda}$, write $\lambda$ for the union $\coprod_{i \in I} \lambda^{(i)}$. We will identify $\boldsymbol{\lambda}$ with the corresponding $I$-labelled Young diagram of shape $\lambda$. This is the unique $I$-labelled Young diagram in which the labels from left to right across a row increase by 1 at each step, and $\lambda^{(i)}$ is the subpartition formed by all rows starting with the label $i$. (Two I-labelled Young diagrams which differ by permuting rows of equal length are considered the same.)

Example 2.1. Suppose $n=3$. Consider the multisegment

$$
[2 ; 2) \oplus[0 ; 2) \oplus[1 ; 4) \oplus[1 ; 1) \oplus[0 ; 2) .
$$

The corresponding $\boldsymbol{\lambda}$ has $\lambda^{(0)}=\left(2^{2}\right), \lambda^{(1)}=(41)$, and $\lambda^{(2)}=(2)$. So

$$
\lambda=\left(42^{3} 1\right), \text { and } \boldsymbol{\lambda}=\begin{array}{|l|l|l|l|}
\hline & 2 & 0 & 1 \\
\hline 0 & 1 & \\
\hline 0 & 1 & \\
\hline 2 & 0 & \\
\hline 1 & &
\end{array} .
$$

The dimension vector of a nilpotent representation in the class corresponding to $\boldsymbol{\lambda}$ is $\mathbf{d}(\boldsymbol{\lambda})=\left(d_{i}(\boldsymbol{\lambda})\right)_{i \in I}$, where $d_{i}(\boldsymbol{\lambda})$ is the number of boxes labelled $i$. Consequently, the $G_{V}$-orbits in $\mathcal{N}_{V}$ are in bijection with $\boldsymbol{\Lambda}(\mathbf{d})=\{\boldsymbol{\lambda} \in \boldsymbol{\Lambda} \mid \mathbf{d}(\boldsymbol{\lambda})=\mathbf{d}\}$. For $\boldsymbol{\lambda} \in \boldsymbol{\Lambda}(\mathbf{d})$, write $\mathcal{O}_{\boldsymbol{\lambda}}$ for the corresponding orbit. In other words,

$$
\mathcal{O}_{\boldsymbol{\lambda}}=\left\{x \in \mathcal{N}_{V} \mid \operatorname{dim} \operatorname{ker} x^{k}=\mathbf{d}\left(\boldsymbol{\lambda}^{\leq k}\right)\right\},
$$

where $\boldsymbol{\lambda}^{\leq k}$ is obtained from $\boldsymbol{\lambda}$ by deleting all but the first $k$ columns. Clearly the $G L(V)$-orbit containing $\mathcal{O}_{\boldsymbol{\lambda}}$ is $\mathcal{O}_{\lambda}$.

We define a partial order $\leq$ on $\boldsymbol{\Lambda}(\mathbf{d})$ by

$$
\boldsymbol{\mu} \leq \boldsymbol{\lambda} \Leftrightarrow \mathcal{O}_{\boldsymbol{\mu}} \subseteq \overline{\mathcal{O}_{\lambda}}
$$

We will be mainly interested in the sub-poset $\Lambda^{\leq 2}(\mathbf{d})$ of $\boldsymbol{\Lambda}(\mathbf{d})$ consisting of all $\boldsymbol{\lambda}$ with at most two rows. The corresponding nilpotent 
representations are either indecomposable or the sum of two indecomposables; as elements of $\operatorname{End}(V)$, they have at most two Jordan blocks. Obviously $\boldsymbol{\mu} \in \boldsymbol{\Lambda}^{\leq 2}(\mathbf{d}), \boldsymbol{\mu} \leq \boldsymbol{\lambda} \Rightarrow \boldsymbol{\lambda} \in \boldsymbol{\Lambda}^{\leq 2}(\mathbf{d})$.

Remark 2.2. All of the above remains true for the linear quiver of type $A_{\infty}$, if we take $I=\mathbb{Z}$. In this case $\Lambda^{\leq 2}(\mathbf{d})$ has at most 2 elements, of which the maximal one parametrizes the orbit which is dense in $\mathcal{N}_{V}=E_{V}$. As we will see, $\Lambda^{\leq 2}(\mathbf{d})$ is more interesting for $\Delta_{n}$, but still much simpler to describe than the whole poset $\boldsymbol{\Lambda}(\mathbf{d})$.

\section{Local Intersection Cohomology}

For $\boldsymbol{\lambda} \in \boldsymbol{\Lambda}(\mathbf{d})$, let $\mathcal{H}^{k} I C\left(\overline{\mathcal{O}_{\boldsymbol{\lambda}}}\right)$ be the $k$-th intersection cohomology sheaf of the (usually singular) variety $\overline{\mathcal{O}_{\lambda}}$. As we will see, this vanishes if $k$ is odd. If $\boldsymbol{\mu} \leq \boldsymbol{\lambda}$, let the local IC polynomial $\tilde{K}_{\boldsymbol{\lambda}, \boldsymbol{\mu}}(t) \in \mathbb{N}[t]$ be

$$
\tilde{K}_{\boldsymbol{\lambda}, \boldsymbol{\mu}}(t)=\sum_{k \geq 0} \operatorname{dim} \mathcal{H}_{x_{\boldsymbol{\mu}}}^{2 k} I C\left(\overline{\mathcal{O}_{\boldsymbol{\lambda}}}\right) t^{k}
$$

where $\mathcal{H}_{x_{\mu}}^{2 k} I C\left(\overline{\mathcal{O}_{\boldsymbol{\lambda}}}\right)$ means the stalk at some point $x_{\boldsymbol{\mu}} \in \mathcal{O}_{\boldsymbol{\mu}}$. Trivially we have $\tilde{K}_{\boldsymbol{\lambda}, \boldsymbol{\lambda}}(t)=1$ for all $\boldsymbol{\lambda}$. By basic properties of intersection cohomology, $\tilde{K}_{\boldsymbol{\lambda}, \boldsymbol{\mu}}(t)$ has constant term 1 and satisfies

$$
\operatorname{deg} \tilde{K}_{\boldsymbol{\lambda}, \boldsymbol{\mu}}<\left(\operatorname{codim} \frac{\mathcal{O}_{\boldsymbol{\lambda}}}{\mathcal{O}_{\boldsymbol{\mu}}}\right) / 2 \text {, if } \boldsymbol{\mu}<\boldsymbol{\lambda} \text {. }
$$

If $\boldsymbol{\mu} \not \mathbf{\lambda}$, we set $\tilde{K}_{\boldsymbol{\lambda}, \boldsymbol{\mu}}(t)=0$. The main result of this paper is:

Theorem 3.1. For $\boldsymbol{\lambda} \geq \boldsymbol{\mu}$ in $\boldsymbol{\Lambda}^{\leq 2}(\mathbf{d}), \tilde{K}_{\boldsymbol{\lambda}, \boldsymbol{\mu}}(t)=1$.

In other words, for $\boldsymbol{\lambda} \geq \boldsymbol{\mu}$ in $\boldsymbol{\Lambda}^{\leq 2}(\mathbf{d}), \overline{\mathcal{O}_{\boldsymbol{\lambda}}}$ is rationally smooth at the points of $\mathcal{O}_{\mu}$ (in general, it is not actually smooth there).

If $n=1, \boldsymbol{\Lambda}(\mathbf{d})=\Lambda(d)$ and $\mathcal{O}_{\boldsymbol{\lambda}}=\mathcal{O}_{\lambda}$. Lusztig proved in [6] that

$$
\tilde{K}_{\lambda, \mu}(t)=t^{n(\mu)-n(\lambda)} K_{\lambda, \mu}\left(t^{-1}\right),
$$

where $K_{\lambda, \mu}(\cdot)$ is the Kostka-Foulkes polynomial and $n(\lambda)=\sum(k-1) \lambda_{k}$. So in this case Theorem 3.1 is well known.

Several important results involve the polynomials $\tilde{K}_{\boldsymbol{\lambda}, \boldsymbol{\mu}}(t)$, which is why one wants to compute them. In [7, §11] Lusztig showed that the orbit closures $\overline{\mathcal{O}_{\boldsymbol{\lambda}}}$ could be embedded as open subvarieties of certain affine Schubert varieties of type $A$, which explains why $\mathcal{H}^{k} I C\left(\overline{\mathcal{O}_{\boldsymbol{\lambda}}}\right)=0$ for $k$ odd. It also means that there is an order-preserving injection

$$
\boldsymbol{\Lambda}(\mathbf{d}) \hookrightarrow \tilde{W}: \boldsymbol{\lambda} \mapsto w_{\boldsymbol{\lambda}},
$$

where $\tilde{W}$ is the affine Weyl group of type $\widetilde{A_{d-1}}$, such that $\tilde{K}_{\boldsymbol{\lambda}, \boldsymbol{\mu}}(t)$ is the affine Kazhdan-Lusztig polynomial $P_{w_{\mu}, w_{\boldsymbol{\lambda}}}(t)$. (This gives a combinatorial algorithm for computing $\tilde{K}_{\boldsymbol{\lambda}, \boldsymbol{\mu}}(t)$ in general, but it is a highly 
impractical one.) So Theorem 3.1 assserts that certain very special affine Kazhdan-Lusztig polynomials are trivial. One can also interpret $\left(\tilde{K}_{\boldsymbol{\lambda}, \boldsymbol{\mu}}(t)\right)$ as the transition matrix between two bases of the generic Hall algebra of $\Delta_{n}$, an algebra of great importance in the theory of quantum affine $\mathfrak{s l}_{n}$ (see for instance [5, §3]). We will not say anything further about either of these points of view.

There are also well-known interpretations of (some of) the values $\tilde{K}_{\boldsymbol{\lambda}, \boldsymbol{\mu}}(1)$ in complex representation theory. In the case $n=1, \tilde{K}_{\lambda, \mu}(1)$ is the Kostka number $K_{\lambda, \mu}$, which gives the multiplicity of the simple representation $V_{\lambda}$ of the symmetric group $S_{d}$ in the induced representa-

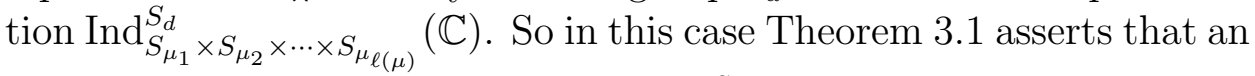
induced representation of the form $\operatorname{Ind}_{S_{\mu_{1}} \times S_{\mu_{2}}}^{S_{d}}(\mathbb{C})$ is multiplicity-free, which is again well known.

If on the other hand $n \geq 2$, let $\zeta \in \mathbb{C}^{\times}$be a primitive $n$-th root of unity. Let $s \in G L(V)$ be the semisimple element which acts by the scalar $\zeta^{-i}$ on $V_{i}$. Then $E_{V}=\{x \in \operatorname{End}(V) \mid \operatorname{Ad}(s)(x)=\zeta x\}$. Thus for any $x \in \mathcal{N}_{V}$, we have an associated standard module $M_{s, x, \zeta}$ of $\widetilde{\mathcal{H}}_{d}$, the affine Hecke algebra of $G L_{d}(\mathbb{C})$ mentioned in the Introduction. Up to isomorphism, this depends only on the $G_{V}$-orbit of $x$, so we will write it as $M_{\boldsymbol{\lambda}}$ for $\boldsymbol{\lambda} \in \boldsymbol{\Lambda}(\mathbf{d})$.

Let $L_{\boldsymbol{\lambda}}$ be the quotient of $M_{\boldsymbol{\lambda}}$ defined in [1], Chapter 8], which is either 0 or a simple module. We say that $\boldsymbol{\lambda} \in \boldsymbol{\Lambda}(\mathbf{d})$ is aperiodic if for all $m$ there exists some $i \in I$ such that $m$ is not a part of $\lambda^{(i)}$. It follows from results of Lusztig that $L_{\boldsymbol{\lambda}} \neq 0$ iff $\boldsymbol{\lambda}$ is aperiodic (see [5, $\S 2$ and Appendix]). All simple constituents of $M_{\boldsymbol{\mu}}$ for $\boldsymbol{\mu} \in \boldsymbol{\Lambda}(\mathbf{d})$ are isomorphic to some such nonzero $L_{\boldsymbol{\lambda}}$, and we have the following multiplicity formula due to Ginzburg ([1, Theorem 8.6.15], [5, §2]):

$$
\left[M_{\boldsymbol{\mu}}: L_{\boldsymbol{\lambda}}\right]=\tilde{K}_{\boldsymbol{\lambda}, \boldsymbol{\mu}}(1) \text {. }
$$

So Theorem 3.1 implies that if $\boldsymbol{\mu} \in \boldsymbol{\Lambda}^{\leq 2}(\mathbf{d}), M_{\boldsymbol{\mu}}$ is multiplicity-free. In fact, this is equivalent to Theorem 3.1 (for $n \geq 2$ ), since an element of $\Lambda^{\leq 2}(\mathbf{d})$ must be aperiodic, unless $n=2$, all $d_{i}$ are equal, and it is the minimal element of $\boldsymbol{\Lambda}^{\leq 2}(\mathbf{d})$. As mentioned in the Introduction, this equivalent formulation is part of the main result of [2]. Thus Theorem 3.1 has already been proved representation-theoretically; we will prove it geometrically in the course of subsequent sections.

\section{Resolutions of the Orbit Closures}

The key to our approach is the fact that each orbit closure $\overline{\mathcal{O}_{\lambda}}$ has an obvious resolution of singularities. Fix $\boldsymbol{\lambda} \in \boldsymbol{\Lambda}(\mathbf{d})$. Let $l=\ell\left(\lambda^{\prime}\right)$ be the number of columns of $\boldsymbol{\lambda}$. Recall that $\boldsymbol{\lambda}^{\leq k} \in \boldsymbol{\Lambda}$ is obtained by 
deleting all but the first $k$ columns of $\boldsymbol{\lambda}$. Define the $I$-graded partial flag variety

$$
\mathcal{F}_{\boldsymbol{\lambda}}=\left\{0=W^{(0)} \subset W^{(1)} \subset \cdots \subset W^{(l)}=V \mid \operatorname{dim} W^{(k)}=\mathbf{d}\left(\boldsymbol{\lambda}^{\leq k}\right)\right\},
$$

and let

$$
\widetilde{\widetilde{\mathcal{O}_{\boldsymbol{\lambda}}}}=\left\{\left(x,\left(W^{(k)}\right)\right) \in \mathcal{N}_{V} \times \mathcal{F}_{\boldsymbol{\lambda}} \mid x\left(W^{(k)}\right) \subseteq W^{(k-1)}, 1 \leq k \leq l\right\} .
$$

In the case $n=1$, this is the cotangent bundle of $\mathcal{F}_{\boldsymbol{\lambda}}=\mathcal{F}_{\lambda}$. In general we have:

Lemma 4.1. $\quad$ 1. $\mathcal{F}_{\boldsymbol{\lambda}}$ is a smooth irreducible projective variety, and

$$
\operatorname{dim} \mathcal{F}_{\boldsymbol{\lambda}}=\sum_{i \in I}\left[\left(\begin{array}{c}
d_{i} \\
2
\end{array}\right)-\sum_{k=1}^{l}\left(\begin{array}{c}
d_{i}\left(\boldsymbol{\lambda}^{\leq k}\right)-d_{i}\left(\boldsymbol{\lambda}^{\leq k-1}\right) \\
2
\end{array}\right)\right] .
$$

2. The second projection $\widetilde{\mathcal{O}_{\boldsymbol{\lambda}}} \rightarrow \mathcal{F}_{\boldsymbol{\lambda}}$ is a vector bundle, with fibres of dimension

$$
\sum_{i \in I}\left[\left(\begin{array}{c}
d_{i} \\
2
\end{array}\right)+\sum_{k=1}^{l}\left(\begin{array}{c}
d_{i}\left(\boldsymbol{\lambda}^{\leq k}\right)-d_{i}\left(\boldsymbol{\lambda}^{\leq k-1}\right)+1 \\
2
\end{array}\right)\right]-\epsilon(\boldsymbol{\lambda}),
$$

where

$$
\begin{aligned}
\epsilon(\boldsymbol{\lambda}) & =\sum_{\text {rows } R \text { of } \boldsymbol{\lambda}} d_{\text {last label of } R}\left(\boldsymbol{\lambda}^{\leq|R|}\right) \\
& =\sum_{i \in I} \sum_{p=1}^{\ell\left(\lambda^{(i)}\right)} d_{i+\lambda_{p}^{(i)}-1}\left(\boldsymbol{\lambda}^{\leq \lambda_{p}^{(i)}}\right) .
\end{aligned}
$$

3. The first projection $p_{\boldsymbol{\lambda}}: \widetilde{\widetilde{\mathcal{O}_{\boldsymbol{\lambda}}}} \rightarrow \mathcal{N}_{V}$ has image $\overline{\mathcal{O}_{\boldsymbol{\lambda}}}$ and is an isomorphism over $\mathcal{O}_{\boldsymbol{\lambda}}$. Hence $p_{\boldsymbol{\lambda}}$ is a resolution of $\overline{\mathcal{O}_{\boldsymbol{\lambda}}}$, and

$$
\begin{aligned}
\operatorname{dim} \mathcal{O}_{\boldsymbol{\lambda}} & =\operatorname{dim} \overline{\mathcal{O}_{\boldsymbol{\lambda}}}=\operatorname{dim} \widetilde{\widetilde{\mathcal{O}_{\boldsymbol{\lambda}}}} \\
& =\sum_{i \in I} d_{i}^{2}-\epsilon(\boldsymbol{\lambda})
\end{aligned}
$$

So if $\boldsymbol{\mu} \leq \boldsymbol{\lambda}, \operatorname{codim} \frac{\mathcal{O}_{\boldsymbol{\lambda}}}{\mathcal{O}_{\boldsymbol{\mu}}}=\epsilon(\boldsymbol{\mu})-\epsilon(\boldsymbol{\lambda})$.

Proof. (1) is obvious, because $\mathcal{F}_{\boldsymbol{\lambda}}$ is the product of partial flag varieties in each $V_{i}$ of the required dimension. Clearly $\widetilde{\widetilde{\mathcal{O}_{\boldsymbol{\lambda}}}} \rightarrow \mathcal{F}_{\boldsymbol{\lambda}}$ is a vector bundle, and the fibre over $\left(W^{(k)}\right) \in \mathcal{F}_{\boldsymbol{\lambda}}$ is

$$
\bigoplus_{i \in I}\left\{\phi \in \operatorname{Hom}\left(V_{i+1}, V_{i}\right) \mid \phi\left(W_{i+1}^{(k)}\right) \subseteq W_{i}^{(k-1)}, 1 \leq k \leq l\right\}
$$


which has dimension

$$
\sum_{i \in I} \sum_{k=1}^{l}\left(d_{i+1}\left(\boldsymbol{\lambda}^{\leq k}\right)-d_{i+1}\left(\boldsymbol{\lambda}^{\leq k-1}\right)\right) d_{i}\left(\boldsymbol{\lambda}^{\leq k-1}\right) .
$$

This is the number of ordered pairs of boxes $\left(b, b^{\prime}\right)$ in $\boldsymbol{\lambda}$ where the column containing $b^{\prime}$ is further right than the column containing $b$, and the label of $b^{\prime}$ is one more than the label of $b$. Considering the box $b^{\prime \prime}$ immediately to the left of $b^{\prime}$, we see that this is the same as the number of ordered pairs of boxes $\left(b, b^{\prime \prime}\right)$ with the same label where the column containing $b^{\prime \prime}$ is further right than or equal to the column containing $b$, and where $b^{\prime \prime}$ is not at the end of a row. This gives (2). For (3), $p_{\boldsymbol{\lambda}}$ is proper since $\mathcal{F}_{\boldsymbol{\lambda}}$ is projective. From the definitions,

$$
p_{\boldsymbol{\lambda}}^{-1}\left(\mathcal{O}_{\boldsymbol{\lambda}}\right)=\left\{\left(x,\left(W^{(k)}\right)\right) \in \mathcal{N}_{V} \times \mathcal{F}_{\boldsymbol{\lambda}} \mid \operatorname{ker} x^{k}=W^{(k)}, 1 \leq k \leq l\right\},
$$

which is clearly open in $\widetilde{\mathcal{O}_{\boldsymbol{\lambda}}}$ and isomorphic to $\mathcal{O}_{\boldsymbol{\lambda}}$. The rest follows since we know from (1) and (2) that $\widetilde{\mathcal{O}_{\boldsymbol{\lambda}}}$ is smooth and irreducible.

Note that $\sum_{i \in I} d_{i}^{2}=\operatorname{dim} G_{V}$. So (3) implies that for $x_{\boldsymbol{\lambda}} \in \mathcal{O}_{\boldsymbol{\lambda}}$, $\operatorname{dim} Z_{G_{V}}\left(x_{\boldsymbol{\lambda}}\right)=\epsilon(\boldsymbol{\lambda})$. (Thus the notation agrees with [5, §3].) Since $Z_{G_{V}}\left(x_{\boldsymbol{\lambda}}\right)$ is an open subvariety of the algebra of endomorphisms of $x_{\boldsymbol{\lambda}}$ as a representation of $\Delta_{n}$, it is connected. So the only $G_{V}$-equivariant simple perverse sheaves on $\mathcal{N}_{V}$ are the shifted intersection cohomology complexes $I C\left(\overline{\mathcal{O}_{\boldsymbol{\lambda}}}\right)\left[\operatorname{dim} \mathcal{O}_{\boldsymbol{\lambda}}\right]$, for $\boldsymbol{\lambda} \in \boldsymbol{\Lambda}(\mathbf{d})$. By the Equivariant Decomposition Theorem (see for example [1, Theorem 8.4.7]),

$$
R\left(p_{\boldsymbol{\lambda}}\right)_{*} \mathbb{C}\left[\operatorname{dim} \mathcal{O}_{\boldsymbol{\lambda}}\right] \cong \bigoplus_{\substack{\boldsymbol{\nu} \leq \boldsymbol{\lambda} \\ j \in \mathbb{Z}}} I C\left(\overline{\mathcal{O}_{\boldsymbol{\nu}}}\right)\left[\operatorname{dim} \mathcal{O}_{\boldsymbol{\nu}}+j\right]^{\oplus a_{\boldsymbol{\lambda}, \boldsymbol{\nu}, j}}
$$

for some $a_{\boldsymbol{\lambda}, \boldsymbol{\nu}, j} \in \mathbb{N}$. Since the left-hand side is Verdier self-dual, $a_{\boldsymbol{\lambda}, \boldsymbol{\nu}, j}=a_{\boldsymbol{\lambda}, \boldsymbol{\nu},-j}$. Since $p_{\boldsymbol{\lambda}}$ is an isomorphism over $\mathcal{O}_{\boldsymbol{\lambda}}, a_{\boldsymbol{\lambda}, \boldsymbol{\lambda}, j}$ is 1 if $j=0$ and 0 otherwise.

Taking stalk at $x_{\boldsymbol{\mu}} \in \mathcal{O}_{\boldsymbol{\mu}}$ of both sides of (4.1), we get

$$
\sum_{k \geq 0} \operatorname{dim} H^{k}\left(p_{\boldsymbol{\lambda}}^{-1}\left(x_{\boldsymbol{\mu}}\right)\right) t^{k}=\sum_{\boldsymbol{\lambda} \geq \boldsymbol{\nu} \geq \boldsymbol{\mu}}\left(\sum_{j \in \mathbb{Z}} a_{\boldsymbol{\lambda}, \boldsymbol{\nu}, j} t^{\epsilon(\boldsymbol{\nu})-\epsilon(\boldsymbol{\lambda})-j}\right) \tilde{K}_{\boldsymbol{\nu}, \boldsymbol{\mu}}\left(t^{2}\right) .
$$

Now

$$
p_{\boldsymbol{\lambda}}^{-1}\left(x_{\boldsymbol{\mu}}\right) \cong\left\{\left(W^{(k)}\right) \in \mathcal{F}_{\boldsymbol{\lambda}} \mid x_{\boldsymbol{\mu}}\left(W^{(k)}\right) \subseteq W^{(k-1)}, 1 \leq k \leq l\right\} .
$$

It is easy to prove (say by induction on $l$ ) that $p_{\boldsymbol{\lambda}}^{-1}\left(x_{\boldsymbol{\mu}}\right)$ has a paving by affine spaces, and hence has no odd cohomologies. (In the cases we use below we will see this another way.) So in fact $a_{\boldsymbol{\lambda}, \boldsymbol{\nu}, j}=0$ if $j$ is of 
opposite parity to $\epsilon(\boldsymbol{\nu})-\epsilon(\boldsymbol{\lambda})$, and if we write $g_{\boldsymbol{\lambda}, \boldsymbol{\mu}}(t)$ for the Poincaré polynomial $\sum_{k \geq 0} \operatorname{dim} H^{2 k}\left(p_{\boldsymbol{\lambda}}^{-1}\left(x_{\boldsymbol{\mu}}\right)\right) t^{k}$, we have

$$
g_{\boldsymbol{\lambda}, \boldsymbol{\mu}}(t)=\sum_{\boldsymbol{\lambda} \geq \boldsymbol{\nu} \geq \boldsymbol{\mu}}\left(\sum_{j \equiv \epsilon(\boldsymbol{\nu})-\epsilon(\boldsymbol{\lambda}) \bmod 2} a_{\boldsymbol{\lambda}, \boldsymbol{\nu}, j} t^{(\epsilon(\boldsymbol{\nu})-\epsilon(\boldsymbol{\lambda})-j) / 2}\right) \tilde{K}_{\boldsymbol{\nu}, \boldsymbol{\mu}}(t) .
$$

Thanks to the degree constraint and others we have mentioned, knowing $g_{\boldsymbol{\lambda}, \boldsymbol{\mu}}(t)$ for all $\boldsymbol{\lambda} \geq \boldsymbol{\mu}$ determines all $a_{\boldsymbol{\lambda}, \boldsymbol{\mu}, j}$ and $\tilde{K}_{\boldsymbol{\lambda}, \boldsymbol{\mu}}(t)$. In fact:

Lemma 4.2. If $b_{\boldsymbol{\lambda}, \boldsymbol{\mu}, j} \in \mathbb{C}$ and $L_{\boldsymbol{\lambda}, \boldsymbol{\mu}}(t) \in \mathbb{C}[t]$ for all $\boldsymbol{\lambda} \geq \boldsymbol{\mu} \in \boldsymbol{\Lambda}(\mathbf{d})$ and $j \in \mathbb{Z}$ satisfy:

1. for all $\boldsymbol{\lambda} \geq \boldsymbol{\mu}$,

$$
g_{\boldsymbol{\lambda}, \boldsymbol{\mu}}(t)=\sum_{\boldsymbol{\lambda} \geq \boldsymbol{\nu} \geq \boldsymbol{\mu}}\left(\sum_{j \equiv \epsilon(\boldsymbol{\nu})-\epsilon(\boldsymbol{\lambda}) \bmod 2} b_{\boldsymbol{\lambda}, \boldsymbol{\nu}, j} t^{(\epsilon(\boldsymbol{\nu})-\epsilon(\boldsymbol{\lambda})-j) / 2}\right) L_{\boldsymbol{\nu}, \boldsymbol{\mu}}(t),
$$

2. $b_{\boldsymbol{\lambda}, \boldsymbol{\lambda}, j}=\delta_{j, 0}$,

3. $b_{\boldsymbol{\lambda}, \boldsymbol{\mu}, j}=b_{\boldsymbol{\lambda}, \boldsymbol{\mu},-j}$,

4. $b_{\boldsymbol{\lambda}, \boldsymbol{\mu}, j}=0$ if $j$ is of opposite parity to $\epsilon(\boldsymbol{\mu})-\epsilon(\boldsymbol{\lambda})$,

5. $L_{\boldsymbol{\lambda}, \boldsymbol{\lambda}}(t)=1$, and

6. $\operatorname{deg} L_{\boldsymbol{\lambda}, \boldsymbol{\mu}}<(\epsilon(\boldsymbol{\mu})-\epsilon(\boldsymbol{\lambda})) / 2$ for $\boldsymbol{\mu}<\boldsymbol{\lambda}$,

then $b_{\boldsymbol{\lambda}, \boldsymbol{\mu}, j}=a_{\boldsymbol{\lambda}, \boldsymbol{\mu}, j}, L_{\boldsymbol{\lambda}, \boldsymbol{\mu}}(t)=\tilde{K}_{\boldsymbol{\lambda}, \boldsymbol{\mu}}(t)$ for all $\boldsymbol{\lambda} \geq \boldsymbol{\mu}, j \in \mathbb{Z}$.

Proof. We know that $b_{\boldsymbol{\lambda}, \boldsymbol{\mu}, j}=a_{\boldsymbol{\lambda}, \boldsymbol{\mu}, j}, L_{\boldsymbol{\lambda}, \boldsymbol{\mu}}(t)=\tilde{K}_{\boldsymbol{\lambda}, \boldsymbol{\mu}}(t)$ is a solution of (1)-(6). We now give an algorithm to determine $b_{\boldsymbol{\lambda}, \boldsymbol{\mu}, j}$ and $L_{\boldsymbol{\lambda}, \boldsymbol{\mu}}(t)$ from (1)-(6), assuming that all $g_{\boldsymbol{\lambda}, \boldsymbol{\mu}}(t)$ are known, thus showing that this is the unique solution. This algorithm is by induction on $\epsilon(\boldsymbol{\mu})-\epsilon(\boldsymbol{\lambda})$. If this is $0, \boldsymbol{\mu}=\boldsymbol{\lambda}$, and $b_{\boldsymbol{\lambda}, \boldsymbol{\lambda}, j}$ and $L_{\boldsymbol{\lambda}, \boldsymbol{\lambda}}(t)$ are determined by (2) and (5) respectively. Otherwise, we can apply the induction hypothesis to $\boldsymbol{\lambda}>\boldsymbol{\nu}$ and $\boldsymbol{\nu}>\boldsymbol{\mu}$, and hence assume that all terms in the right-hand side of (1) have been determined except $\boldsymbol{\nu}=\boldsymbol{\lambda}$ and $\boldsymbol{\nu}=\boldsymbol{\mu}$. So we know the value of

$$
L_{\boldsymbol{\lambda}, \boldsymbol{\mu}}(t)+\sum_{j \equiv \epsilon(\boldsymbol{\mu})-\epsilon(\boldsymbol{\lambda}) \bmod 2} b_{\boldsymbol{\lambda}, \boldsymbol{\mu}, j} t^{(\epsilon(\boldsymbol{\mu})-\epsilon(\boldsymbol{\lambda})-j) / 2} .
$$

Because of (6), this determines $b_{\boldsymbol{\lambda}, \boldsymbol{\mu}, j}$ for $j \leq 0, j \equiv \epsilon(\boldsymbol{\mu})-\epsilon(\boldsymbol{\lambda}) \bmod 2$, hence for all $j$ by (3) and (4). Thus $L_{\boldsymbol{\lambda}, \boldsymbol{\mu}}(t)$ also is determined.

The argument of this proof is familiar in other contexts, for instance in the study of Kazhdan-Lusztig polynomials.

Remark 4.3. Note that if we want to determine a specific polynomial $\tilde{K}_{\boldsymbol{\lambda}, \boldsymbol{\mu}}(t)$ by this method, we only need to know $g_{\boldsymbol{\rho}, \boldsymbol{\nu}}(t)$ for those $\boldsymbol{\rho}, \boldsymbol{\nu}$ such that $\boldsymbol{\lambda} \geq \boldsymbol{\rho} \geq \boldsymbol{\nu} \geq \boldsymbol{\mu}$; that is, we can restrict attention to the interval 
$[\boldsymbol{\mu}, \boldsymbol{\lambda}] \subseteq \boldsymbol{\Lambda}(\mathbf{d})$. In particular, in proving Theorem 3.1 we can restrict attention to $\boldsymbol{\Lambda}^{\leq 2}(\mathbf{d})$. In the remaining sections, we will calculate $g_{\boldsymbol{\lambda}, \boldsymbol{\mu}}(t)$ for $\boldsymbol{\lambda} \geq \boldsymbol{\mu}$ in $\boldsymbol{\Lambda}^{\leq 2}(\mathbf{d})$ and show that it has the required form.

Remark 4.4. Note that in the case $n=1, p_{\lambda}: \widetilde{\overline{\mathcal{O}_{\lambda}}} \rightarrow \overline{\mathcal{O}_{\lambda}}$ is semismall; equivalently, $a_{\lambda, \nu, j}=0$ if $j \neq 0$. For example, $p_{(d)}$ is the famous Springer resolution of the nilpotent cone. The Poincaré polynomial

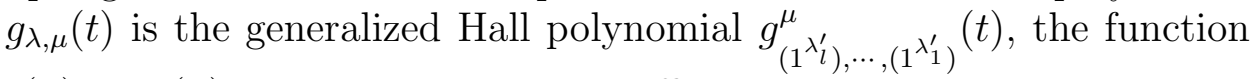
$\epsilon(\lambda)$ is $2 n(\lambda)+d$, and the nonzero coefficient $a_{\lambda, \nu, 0}$ is the Kostka number $K_{\nu^{\prime}, \lambda^{\prime}}$ (see 88, III.6, Example 5]). This can be proved geometrically, using Fourier transform (see [3, §5]). When $n \geq 2$, Fourier transform does not seem to be useful for analysing $R\left(p_{\boldsymbol{\lambda}}\right)_{*} \mathbb{C}$.

Remark 4.5. An example when $p_{\boldsymbol{\lambda}}$ is not semismall is the case $n=2$, $\boldsymbol{\lambda}=[0 ; 4) \oplus[0 ; 1)$. If $\boldsymbol{\mu}=[0 ; 2) \oplus[0 ; 2) \oplus[0 ; 1)$, the reader can check that $a_{\boldsymbol{\lambda}, \boldsymbol{\mu}, 1}=a_{\boldsymbol{\lambda}, \boldsymbol{\mu},-1}=1$.

Remark 4.6. When $n=2$, it is easy to see that

$$
\epsilon(\boldsymbol{\lambda})=\frac{\left(d_{0}-d_{1}\right)^{2}+\epsilon(\lambda)}{2},
$$

whence $\operatorname{dim} \mathcal{O}_{\boldsymbol{\lambda}}=\frac{1}{2} \operatorname{dim} \mathcal{O}_{\lambda}$. This is well known, since the orbits $\mathcal{O}_{\boldsymbol{\lambda}}$ are the nilpotent orbits of the symmetric pair $\left(\mathfrak{g l}_{d}, \mathfrak{g l}_{d_{0}} \oplus \mathfrak{g l}_{d_{1}}\right)$.

Remark 4.7. When $n \geq 3$, it is not true in general that $\epsilon(\boldsymbol{\lambda})$ only depends on $\mathbf{d}$ and $\lambda$. (Consider $n=3, d_{0}=2, d_{1}=d_{2}=1, \lambda=\left(21^{2}\right)$.) However, it is still true that $\boldsymbol{\mu} \leq \boldsymbol{\lambda}, \mu=\lambda \Rightarrow \boldsymbol{\mu}=\boldsymbol{\lambda}$. This can be seen by interpreting $\overline{\mathcal{O}_{\boldsymbol{\lambda}}}$ as the image of $p_{\boldsymbol{\lambda}}$.

\section{Preliminary Lemmas}

Now we restrict attention to $\boldsymbol{\lambda} \in \boldsymbol{\Lambda}^{\leq 2}(\mathbf{d})$ and the corresponding nilpotent orbits. It is convenient to return to multisegment notation, and write elements of $\boldsymbol{\Lambda}^{\leq 2}(\mathbf{d})$ as $\left[i_{1} ; l_{1}\right) \oplus\left[i_{2} ; l_{2}\right)$ where $l_{2}$ is possibly zero (by convention $[i ; 0)$ is the empty partition). For any $m \in \mathbb{Z}$, write $\{m\}$ for the unique element of $\{0,1, \cdots, n-1\} \cap(m+n \mathbb{Z})$. So

$$
\left\lfloor\frac{m}{n}\right\rfloor=\frac{m-\{m\}}{n},\left\lceil\frac{m}{n}\right\rceil=\frac{m+\{-m\}}{n} .
$$


Lemma 5.1. Let $\boldsymbol{\lambda}=\left[i_{1} ; l_{1}\right) \oplus\left[i_{2} ; l_{2}\right)$, where the factors are ordered so that $l_{1} \geq l_{2}$. Then

$$
\begin{aligned}
\epsilon(\boldsymbol{\lambda})=\left\lceil\frac{l_{1}}{n}\right\rceil & +\left\lceil\frac{l_{2}}{n}\right\rceil+\left\lceil\frac{l_{2}-\left\{i_{1}-i_{2}+l_{1}-1\right\}}{n}\right\rceil \\
+ & \left\lceil\frac{l_{2}-\left\{i_{1}-i_{2}\right\}}{n}\right\rceil .
\end{aligned}
$$

Proof. This is trivial from the definition of $\epsilon$.

Fix $\boldsymbol{\lambda}=\left[i_{1} ; l_{1}\right) \oplus\left[i_{2} ; l_{2}\right)$, with $l_{1} \geq l_{2} \geq 0$. Let

$$
\begin{aligned}
\boldsymbol{\Lambda}^{\leq 2}(\mathbf{d})_{\leq \boldsymbol{\lambda}} & =\left\{\boldsymbol{\mu} \in \boldsymbol{\Lambda}^{\leq 2}(\mathbf{d}) \mid \boldsymbol{\mu} \leq \boldsymbol{\lambda}\right\}, \\
\boldsymbol{\Lambda}^{\leq 2}(\mathbf{d})_{\leq \boldsymbol{\lambda}}^{i_{1}, i_{2}} & =\left\{\boldsymbol{\mu}=\left[i_{1} ; m_{1}\right) \oplus\left[i_{2} ; m_{2}\right) \mid \boldsymbol{\mu} \leq \boldsymbol{\lambda}\right\} .
\end{aligned}
$$

Lemma 5.2. If $l_{2} \geq 1, \boldsymbol{\Lambda}^{\leq 2}(\mathbf{d})_{\leq \boldsymbol{\lambda}}=\boldsymbol{\Lambda}^{\leq 2}(\mathbf{d})_{\leq \boldsymbol{\lambda}}^{i_{1}, i_{2}}$. If $l_{2}=0$,

$$
\boldsymbol{\Lambda}^{\leq 2}(\mathbf{d})_{\leq \boldsymbol{\lambda}}=\bigcup_{i_{2}} \boldsymbol{\Lambda}^{\leq 2}(\mathbf{d})_{\leq \boldsymbol{\lambda}}^{i_{1}, i_{2}}
$$

and the union is disjoint except for $\boldsymbol{\lambda}$.

Proof. If $l_{2} \geq 1$, we have $\operatorname{dim} \operatorname{ker} x=\left\{i_{1}, i_{2}\right\}$ for any $x \in \mathcal{O}_{\boldsymbol{\lambda}}$, where $\left\{i_{1}, i_{2}\right\}$ is considered as a multiset, and hence $\operatorname{dim}$ ker $x \geq\left\{i_{1}, i_{2}\right\}$ for any $x \in \overline{\mathcal{O}_{\boldsymbol{\lambda}}}$. Thus if $\boldsymbol{\mu} \leq \boldsymbol{\lambda}$ and $\boldsymbol{\mu} \in \boldsymbol{\Lambda}^{\leq 2}(\mathbf{d}), \boldsymbol{\mu}$ must be $\left[i_{1} ; m_{1}\right) \oplus$ $\left[i_{2} ; m_{2}\right)$ for some $m_{1}, m_{2} \in \mathbb{N}$. If $l_{2}=0$, we know only that for $x \in \overline{\mathcal{O}_{\boldsymbol{\lambda}}}$, $\operatorname{dim} \operatorname{ker} x \geq\left\{i_{1}\right\}$. But if $\boldsymbol{\mu}<\boldsymbol{\lambda}$ and $\boldsymbol{\mu} \in \boldsymbol{\Lambda}^{\leq 2}(\mathbf{d}), \boldsymbol{\mu}$ must have two rows, so there is a unique $i_{2}$ such that $\boldsymbol{\mu}=\left[i_{1} ; m_{1}\right) \oplus\left[i_{2} ; m_{2}\right)$.

Lemma 5.3. Suppose $\boldsymbol{\mu}=\left[i_{1} ; m_{1}\right) \oplus\left[i_{2} ; m_{2}\right) \in \Lambda^{\leq 2}(\mathbf{d})_{\leq \boldsymbol{\lambda}}^{i_{1}, i_{2}}$. Then

$$
m_{1}+m_{2}=l_{1}+l_{2}, l_{1} \geq m_{1} \geq l_{2},
$$

and either

$$
\begin{aligned}
& \text { (a) } m_{1} \equiv l_{1}, m_{2} \equiv l_{2} \bmod n ; \text { or } \\
& \text { (b) } m_{1} \equiv i_{2}-i_{1}+l_{2}, m_{2} \equiv i_{1}-i_{2}+l_{1} \bmod n \text {. }
\end{aligned}
$$

(It is possible that (a) and (b) both hold.)

Proof. That $m_{1}+m_{2}=d=l_{1}+l_{2}$ is obvious. Since $\mathcal{O}_{\boldsymbol{\mu}} \subseteq \overline{\mathcal{O}_{\boldsymbol{\lambda}}}$, we must have $\mathcal{O}_{\mu} \subseteq \overline{\mathcal{O}_{\lambda}}$, which implies that $l_{1} \geq m_{1} \geq l_{2}$. Finally,

$$
\mathbf{d}\left(\left[i_{1} ; m_{1}\right) \oplus\left[i_{2} ; m_{2}\right)\right)=\mathbf{d}\left(\left[i_{1} ; l_{1}\right) \oplus\left[i_{2} ; l_{2}\right)\right) \text {. }
$$

Subtracting $\mathbf{d}\left(\left[i_{1} ; m_{1}\right) \oplus\left[i_{2} ; l_{2}\right)\right)$ from both sides, we get

$$
\mathbf{d}\left(\left[i_{2}+l_{2} ; m_{2}-l_{2}\right)\right)=\mathbf{d}\left(\left[i_{1}+m_{1} ; l_{1}-m_{1}\right)\right) .
$$

This clearly implies either (a) or (b). 
It will be easier to get a converse to this Lemma after we describe the fibres $p_{\boldsymbol{\lambda}}^{-1}\left(x_{\boldsymbol{\mu}}\right)$. For $s_{1}, s_{2} \in \mathbb{N}$, let $\mathcal{B}_{\left(s_{1}, s_{2}\right)}$ be the Springer fibre $p_{\left(s_{1}+s_{2}\right)}^{-1}\left(x_{\left(s_{1}\right) \oplus\left(s_{2}\right)}\right)$, a special case of this fibre when $n=1$. (The choices of the vector space of dimension $s_{1}+s_{2}$ and the element $x_{\left(s_{1}\right) \oplus\left(s_{2}\right)} \in$ $\mathcal{O}_{\left(s_{1}\right) \oplus\left(s_{2}\right)}$ are unimportant.)

Lemma 5.4. Let $\boldsymbol{\mu}=\left[i_{1} ; m_{1}\right) \oplus\left[i_{2} ; m_{2}\right) \neq \boldsymbol{\lambda}$, where $m_{1}$ and $m_{2}$ satisfy the conditions in Lemma 5.9. Then $p_{\boldsymbol{\lambda}}^{-1}\left(x_{\boldsymbol{\mu}}\right) \cong \mathcal{B}_{\left(s_{1}, s_{2}\right)}$, where

$$
s_{1}=\left\lfloor\frac{m_{1}-l_{2}-\left\{i_{2}-i_{1}\right\}}{n}\right\rfloor, s_{2}=\left\lfloor\frac{m_{2}-l_{2}}{n}\right\rfloor,
$$

so that

$$
s_{1}+s_{2}=s:=\frac{l_{1}-l_{2}-\left\{i_{2}-i_{1}\right\}-\left\{i_{1}-i_{2}+l_{1}-l_{2}\right\}}{n} .
$$

(We declare $\mathcal{B}_{\left(-1, s_{2}\right)}$ to be empty.)

Proof. By definition,

$$
p_{\boldsymbol{\lambda}}^{-1}\left(x_{\boldsymbol{\mu}}\right) \cong\left\{\left(W^{(k)}\right) \in \mathcal{F}_{\boldsymbol{\lambda}} \mid x_{\boldsymbol{\mu}}\left(W^{(k)}\right) \subseteq W^{(k-1)}, 1 \leq k \leq l_{1}\right\} .
$$

It is clear that for $1 \leq k \leq l_{2}, W^{(k)}$ is forced to be $\operatorname{ker} x_{\boldsymbol{\mu}}^{k}$. Hence $p_{\boldsymbol{\lambda}}^{-1}\left(x_{\boldsymbol{\mu}}\right) \cong p_{\boldsymbol{\lambda}^{\prime}}^{-1}\left(x_{\boldsymbol{\mu}^{\prime}}\right)$ where

$$
\boldsymbol{\lambda}^{\prime}=\left[i_{1}+l_{2} ; l_{1}-l_{2}\right), \boldsymbol{\mu}^{\prime}=\left[i_{1}+l_{2} ; m_{1}-l_{2}\right) \oplus\left[i_{2}+l_{2} ; m_{2}-l_{2}\right) .
$$

Since $\boldsymbol{\lambda} \neq \boldsymbol{\mu}, m_{2}-l_{2}>0$. In this new fibre, if $i_{1} \neq i_{2}, W^{(1)}$ is forced to be ( $\left.\operatorname{ker} x_{\boldsymbol{\mu}^{\prime}}\right)_{i_{1}+l_{2}}$. Passing to the quotient space and repeating, we see that $W^{(k)}$ is uniquely determined for $1 \leq k \leq\left\{i_{2}-i_{1}\right\}$, so $p_{\boldsymbol{\lambda}^{\prime}}^{-1}\left(x_{\boldsymbol{\mu}^{\prime}}\right) \cong p_{\boldsymbol{\lambda}^{\prime \prime}}^{-1}\left(x_{\boldsymbol{\mu}^{\prime \prime}}\right)$ where

$$
\begin{aligned}
& \boldsymbol{\lambda}^{\prime \prime}=\left[i_{2}+l_{2} ; l_{1}-l_{2}-\left\{i_{2}-i_{1}\right\}\right), \\
& \boldsymbol{\mu}^{\prime \prime}=\left[i_{2}+l_{2} ; m_{1}-l_{2}-\left\{i_{2}-i_{1}\right\}\right) \oplus\left[i_{2}+l_{2} ; m_{2}-l_{2}\right) .
\end{aligned}
$$

(If any lengths become negative here we understand that the corresponding fibre is empty.)

We now wish to apply the dual argument, so note that the end box of $\boldsymbol{\lambda}^{\prime \prime}$ is labelled $i_{1}+l_{1}-1$, and the end labels of $\boldsymbol{\mu}^{\prime \prime}$ are $i_{1}+l_{1}-1$ and $i_{2}+l_{2}-1$ in some order. If $i_{1}+l_{1} \neq i_{2}+l_{2}, W^{\left(l_{1}-l_{2}-\left\{i_{2}-i_{1}\right\}-1\right)}$ is forced to be the preimage of $\left(\operatorname{coker} x_{\boldsymbol{\mu}^{\prime \prime}}\right)_{i_{2}+l_{2}-1}$. Hence $W^{\left(l_{1}-l_{2}-\left\{i_{2}-i_{1}\right\}-k\right)}$ is unique for $1 \leq k \leq\left\{l_{1}-l_{2}+i_{1}-i_{2}\right\}$, so $p_{\boldsymbol{\lambda}^{\prime \prime}}^{-1}\left(x_{\boldsymbol{\mu}^{\prime \prime}}\right) \cong p_{\boldsymbol{\lambda}^{\prime \prime \prime}}^{-1}\left(x_{\boldsymbol{\mu}^{\prime \prime \prime}}\right)$, where

$$
\boldsymbol{\lambda}^{\prime \prime \prime}=\left[i_{2}+l_{2} ; l_{1}-l_{2}-\left\{i_{2}-i_{1}\right\}-\left\{l_{1}-l_{2}+i_{1}-i_{2}\right\}\right)=\left[i_{2}+l_{2} ; n s\right),
$$

and $\boldsymbol{\mu}^{\prime \prime \prime}$ is obtained from $\boldsymbol{\mu}^{\prime \prime}$ by deleting the last $\left\{l_{1}-l_{2}+i_{1}-i_{2}\right\}$ boxes of the row whose end label is $i_{1}+l_{1}-1$. This is the first row if (a) holds and the second row if (b) holds; if both hold, there are no boxes to be deleted. In either case, $\boldsymbol{\mu}^{\prime \prime \prime}=\left[i_{2}+l_{2} ; n s_{1}\right) \oplus\left[i_{2}+l_{2} ; n s_{2}\right)$. 
So in the end it suffices to show that

$$
p_{\left[i ; n\left(s_{1}+s_{2}\right)\right)}^{-1}\left(x_{\left[i ; n s_{1}\right) \oplus\left[i ; n s_{2}\right)}\right) \cong \mathcal{B}_{\left(s_{1}, s_{2}\right)} .
$$

This is obvious, since each $I$-graded flag in the left-hand side is uniquely determined by the induced flag in the subspace corresponding to $i$.

Example 5.5. To illustrate this proof, here is an example of the sequence of steps when $n=3$ (the boxes with bold borders are the ones to be removed):

$$
\begin{aligned}
& \boldsymbol{\lambda}=\begin{array}{|l|l|l|l|l|l|l|l|l|l}
\hline & 1 & 2 & 0 & 1 & 2 & 0 & 1 & 2 & 0
\end{array}, \boldsymbol{\mu}=\begin{array}{|l|l|l|l|l|l|l|l|}
0 & 1 & 2 & 0 & 1 & 2 & 0 \\
\hline 1 & 2 & \multicolumn{10}{|c}{} & 2 & 0 & 1 & 2 & &
\end{array}
\end{aligned}
$$

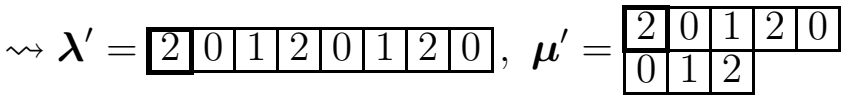

$$
\begin{aligned}
& \rightsquigarrow \boldsymbol{\lambda}^{\prime \prime}=\begin{array}{|l|l|l|l|l|l|l|l|l|l|l|l|}
0 & 1 & 2 & 0 & 1 & 2 & 0
\end{array}, \boldsymbol{\mu}^{\prime \prime}=\begin{array}{lllll}
0 & 1 & 2 & 0 \\
\hline 0 & 1 & 2 & \\
\hline
\end{array} \\
& \rightsquigarrow \boldsymbol{\lambda}^{\prime \prime \prime}=\begin{array}{|l|l|l|l|l|l}
0 & 1 & 2 & 0 & 1 & 2 \\
\hline
\end{array}, \boldsymbol{\mu}^{\prime \prime \prime}=\begin{array}{|l|l|l|}
0 & 1 & 2 \\
\hline 0 & 1 & 2 \\
\hline
\end{array} \rightsquigarrow \mathcal{B}_{(1,1)} .
\end{aligned}
$$

Corollary 5.6. Let $\boldsymbol{\mu}=\left[i_{1} ; m_{1}\right) \oplus\left[i_{2} ; m_{2}\right)$. Then $\boldsymbol{\mu} \in \boldsymbol{\Lambda}^{\leq 2}(\mathbf{d})_{<\boldsymbol{\lambda}}^{i_{1}, i_{2}}$ if and only if

$$
m_{1}+m_{2}=l_{1}+l_{2}, l_{1} \geq m_{1} \geq l_{2}+\left\{i_{2}-i_{1}\right\},
$$

and either (a) or (b) holds.

Proof. We know from Lemma 4.1 that $\overline{\mathcal{O}_{\boldsymbol{\lambda}}}$ is the image of $p_{\boldsymbol{\lambda}}$, so $\boldsymbol{\mu} \leq \boldsymbol{\lambda}$ iff $p_{\boldsymbol{\lambda}}^{-1}\left(x_{\boldsymbol{\mu}}\right)$ is nonempty. Lemma 5.4 shows that for $m_{1}, m_{2}$ as in Lemma 5.3, $p_{\boldsymbol{\lambda}}^{-1}\left(x_{\boldsymbol{\mu}}\right)$ is empty if and only if (a) holds and $m_{1}-l_{2}<\left\{i_{2}-i_{1}\right\}$.

Corollary 5.7. For $\boldsymbol{\mu}<\boldsymbol{\lambda}, p_{\boldsymbol{\lambda}}^{-1}\left(x_{\boldsymbol{\mu}}\right)$ has no odd cohomologies, and

$$
g_{\boldsymbol{\lambda}, \boldsymbol{\mu}}(t)=\sum_{k=0}^{\min \left\{s_{1}, s_{2}\right\}}\left(\left(\begin{array}{l}
s \\
k
\end{array}\right)-\left(\begin{array}{c}
s \\
k-1
\end{array}\right)\right) t^{k},
$$

where $s_{1}, s_{2}$ and $s$ are as in Lemma 5.4.

Proof. Springer fibres such as $\mathcal{B}_{\left(s_{1}, s_{2}\right)}$ have no odd cohomologies. The right-hand side is the Green polynomial $Q_{\left(1^{s}\right)}^{\left(s_{1}, s_{2}\right)}(t)$, which is well known to equal $\sum_{k \geq 0} \operatorname{dim} H^{2 k}\left(\mathcal{B}_{\left(s_{1}, s_{2}\right)}\right) t^{k}$. 


\section{Description of the Posets}

Maintain the notations of $\S 5$. In particular, $\boldsymbol{\lambda}=\left[i_{1} ; l_{1}\right) \oplus\left[i_{2} ; l_{2}\right)$ where $l_{1} \geq l_{2} \geq 0$; and $s$ is as in Lemma 5.4. Using Corollary 5.6, we can completely describe the poset $\boldsymbol{\Lambda}^{\leq 2}(\mathbf{d})_{<\boldsymbol{\lambda}}^{\imath_{1}, i_{2}}$ and hence the poset $\boldsymbol{\Lambda}^{\leq 2}(\mathbf{d})$ as a whole. The arguments are trivial applications of the Lemmas in $\S 5$, and are mostly left to the reader. There are four cases.

Case 1: $i_{1}=i_{2}=i, l_{1} \equiv l_{2} \bmod n$.

In this case conditions (a) and (b) coincide, and $s=\frac{l_{1}-l_{2}}{n}$. If $l_{1}-l_{2}=0$ or $n, \boldsymbol{\lambda}$ is the unique element of $\boldsymbol{\Lambda}^{\leq 2}(\mathbf{d})_{\leq \boldsymbol{\lambda}}^{i, i}$, and conversely. Otherwise, $\left[i ; l_{1}-n\right) \oplus\left[i ; l_{2}+n\right)$ is a predecessor of $\boldsymbol{\lambda}$, also in Case 1 . So $\boldsymbol{\Lambda}^{\leq 2}(\mathbf{d})_{\leq \boldsymbol{\lambda}}^{i, i}$ is a chain:

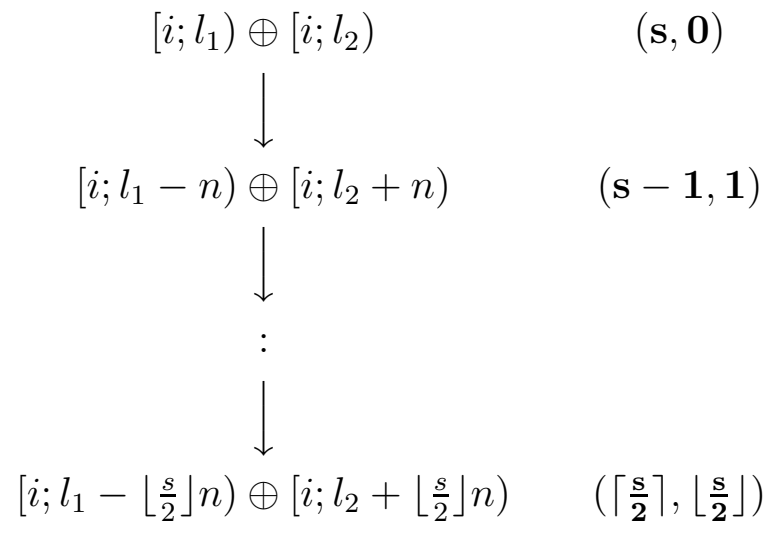

Here and in subsequent diagrams $\boldsymbol{\mu} \rightarrow \boldsymbol{\nu}$ means that $\boldsymbol{\nu}$ is a predecessor of $\boldsymbol{\mu}$, and the boldface labels are the values of $\left(s_{1}, s_{2}\right)$ as in Lemma 5.4 (whose order is actually not determined in the case that $i_{1}=i_{2}$ ). It is easy to see from Lemma 5.1 that $\epsilon\left(\left[i ; l_{1}-n\right) \oplus\left[i ; l_{2}+n\right)\right)=\epsilon(\boldsymbol{\lambda})-2$. So the codimension at each link of the chain is 2 . There are $\left\lfloor\frac{s}{2}\right\rfloor$ links.

Case 2: $i_{1}=i_{2}=i, l_{1} \not \equiv l_{2} \bmod n$.

Here conditions (a) and (b) are mutually exclusive, and $s=\left\lfloor\frac{l_{1}-l_{2}}{n}\right\rfloor$. It is easy to see that $\boldsymbol{\lambda}$ is the unique element of $\boldsymbol{\Lambda}^{\leq 2}(\mathbf{d})_{<\boldsymbol{\lambda}}^{i, i}$ if and only if $l_{1}-l_{2}<n$; otherwise, $\left[i ; l_{1}-\left\{l_{1}-l_{2}\right\}\right) \oplus\left[i ; l_{2}+\left\{l_{1}-l_{2}\right\}\right)$ is a predecessor of $\boldsymbol{\lambda}$, also in Case 2, and satisfying condition (b). Again, $\boldsymbol{\Lambda}^{\leq 2}(\mathbf{d})_{\leq \boldsymbol{\lambda}}^{i, i}$ is 
a chain:

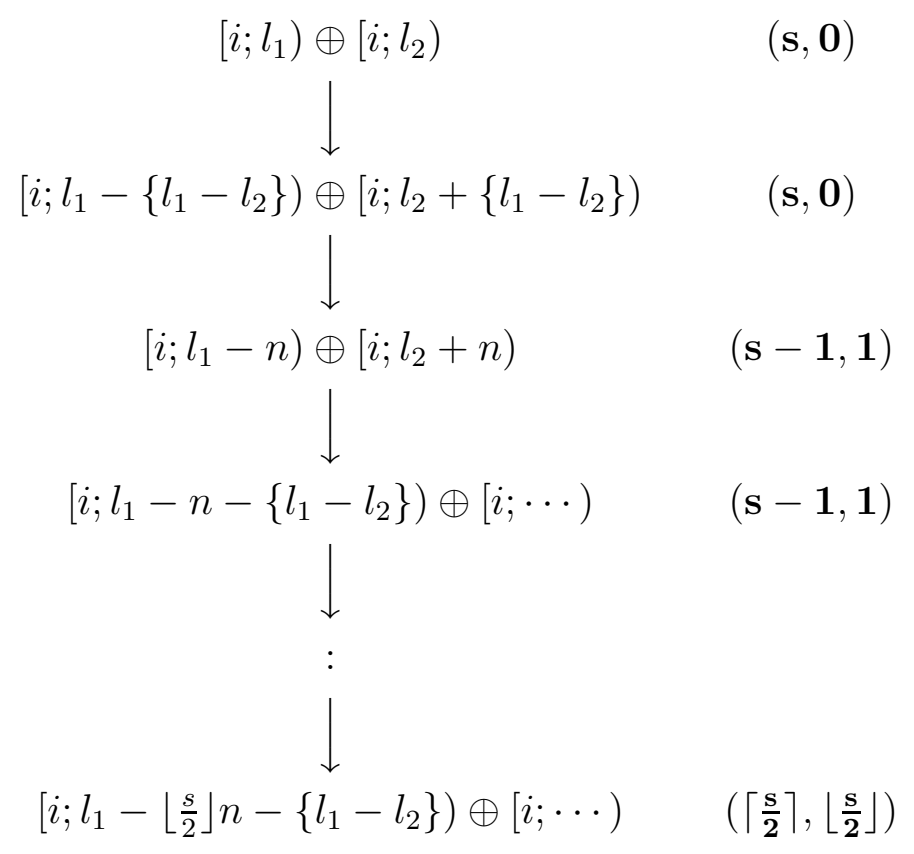

(To make this and later diagrams legible, some row lengths are omitted; recall that for every element the sum of the lengths is $l_{1}+l_{2}$.) If $s$ is even, the minimal element is actually the same as the element which might be thought to be directly above it, namely $\left[i ; l_{1}-\frac{s}{2} n\right) \oplus\left[i ; l_{2}+\frac{s}{2} n\right)$. So there are always $s$ links in the chain. This time the codimension at each link is 1 .

Case 3: $i_{1} \neq i_{2}, l_{1}-l_{2} \equiv i_{2}-i_{1} \bmod n$.

In this case conditions (a) and (b) coincide, and $s=\left\lfloor\frac{l_{1}-l_{2}}{n}\right\rfloor$. The condition for $\boldsymbol{\lambda}$ to be the only element of $\boldsymbol{\Lambda}^{\leq 2}(\mathbf{d})_{\leq \boldsymbol{\lambda}}^{i_{1}, i_{2}}$ is that $l_{1}-l_{2}=$ $\left\{i_{2}-i_{1}\right\}$. Otherwise, $\left[i_{2} ; l_{1}-\left\{l_{1}-l_{2}\right\}\right) \oplus\left[i_{1} ; l_{2}+\left\{l_{1}-l_{2}\right\}\right)$ is a predecessor of $\boldsymbol{\lambda}$, also in Case 3. The summands here are in the right order, i.e. 
$l_{1}-\left\{l_{1}-l_{2}\right\}>l_{2}+\left\{l_{1}-l_{2}\right\}$. Once more $\boldsymbol{\Lambda}^{\leq 2}(\mathbf{d})_{\leq \boldsymbol{\lambda}}^{i_{1}, i_{2}}$ is a chain:

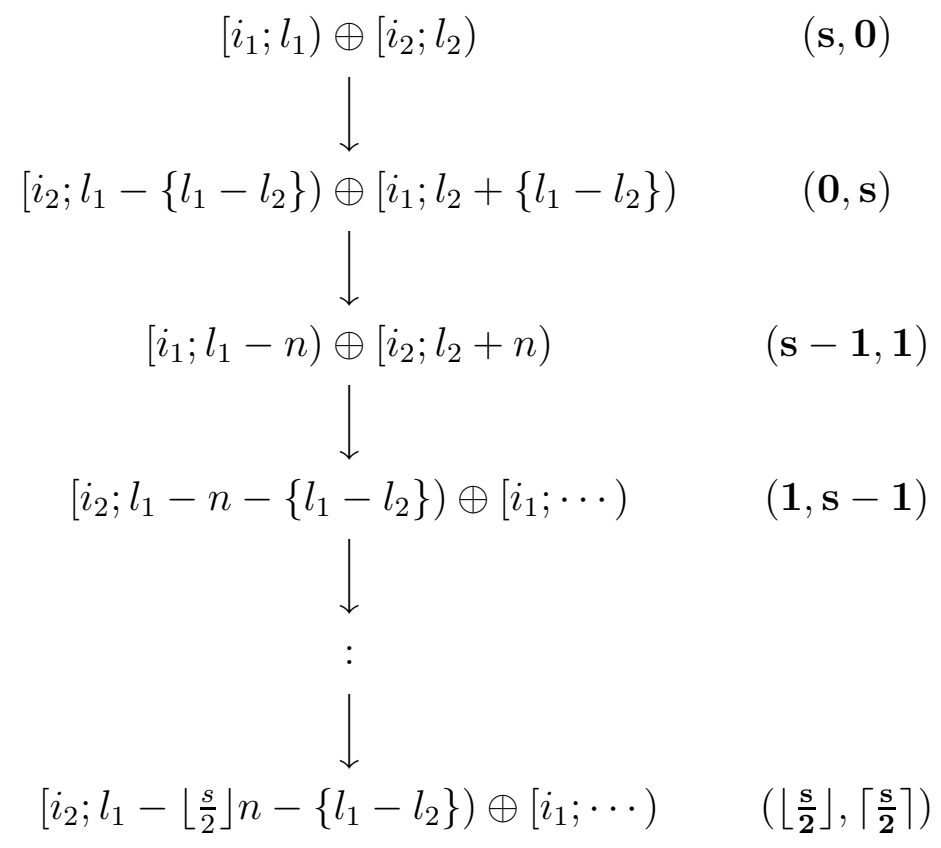

If $s$ is even, the minimal element here is also $\left[i_{1} ; l_{1}-\frac{s}{2} n\right) \oplus\left[i_{2} ; l_{2}+\frac{s}{2} n\right)$, so there are always $s$ links in the chain. Again the codimension at each link is 1 .

Case 4: $i_{1} \neq i_{2}, l_{1}-l_{2} \not \equiv i_{2}-i_{1} \bmod n$.

Conditions (a) and (b) are mutually exclusive, and $s=\left\lfloor\frac{l_{1}-l_{2}}{n}\right\rfloor$ or $\left\lfloor\frac{l_{1}-l_{2}}{n}\right\rfloor-1$ depending on whether $\left\{l_{1}-l_{2}\right\}$ or $\left\{i_{2}-i_{1}\right\}$ is larger. If $l_{1}-l_{2}<\left\{i_{2}-i_{1}\right\}, \boldsymbol{\lambda}$ is the unique element of $\boldsymbol{\Lambda}^{\leq 2}(\mathbf{d})_{<\boldsymbol{\lambda}}^{i_{1}, i_{2}}$, and conversely. Otherwise, write $A=\left\{i_{2}-i_{1}\right\}, B=\left\{i_{1}-i_{2}+\bar{l}_{1}-l_{2}\right\}$; then $\left[i_{1} ; l_{1}-B\right) \oplus\left[i_{2} ; l_{2}+B\right)$ and $\left[i_{2} ; l_{1}-A\right) \oplus\left[i_{1} ; l_{2}+A\right)$ are predecessors of $\boldsymbol{\lambda}$, also in Case 4 , and satisfying condition (b). These coincide iff $l_{1}-l_{2}<n+\left\{i_{2}-i_{1}\right\}$, in which case their common value is a minimal element; otherwise, the summands are in the right order. The poset 
$\Lambda^{\leq 2}(\mathbf{d})_{\leq \boldsymbol{\lambda}}^{i_{1}, i_{2}}$ is as follows:

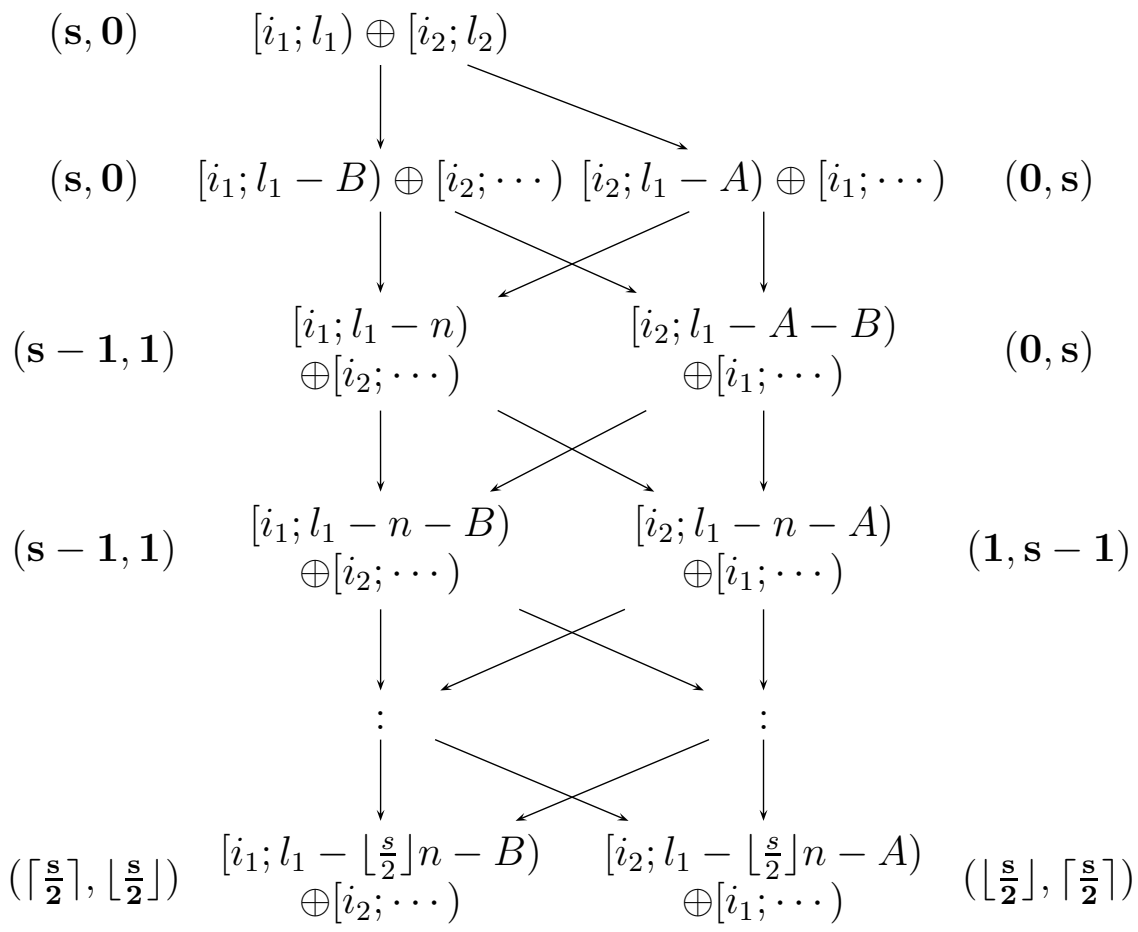

If $s$ is even, the bottom two elements coincide and are minimal. If $s$ is odd, there is one more element below these two, namely

$$
\left[i_{1} ; l_{1}-\left\lceil\frac{s}{2}\right\rceil n\right) \oplus\left\lceil i_{2} ; l_{2}+\left\lceil\frac{s}{2}\right\rceil n\right) \quad\left(\left\lfloor\frac{\mathbf{s}}{\mathbf{2}}\right\rfloor,\left\lceil\frac{\mathbf{s}}{\mathbf{2}}\right\rceil\right) .
$$

So the length of a maximal chain in this poset is $s+1$. Again, all predecessors have codimension 1.

Example 6.1. As an example of how these posets $\boldsymbol{\Lambda}^{\leq 2}(\mathbf{d})_{\leq \boldsymbol{\lambda}}^{i_{1}, i_{2}}$ fit together to make $\boldsymbol{\Lambda}^{\leq 2}(\mathbf{d})$, the following is the case $n=2, d_{0}=d_{1}=3$ :

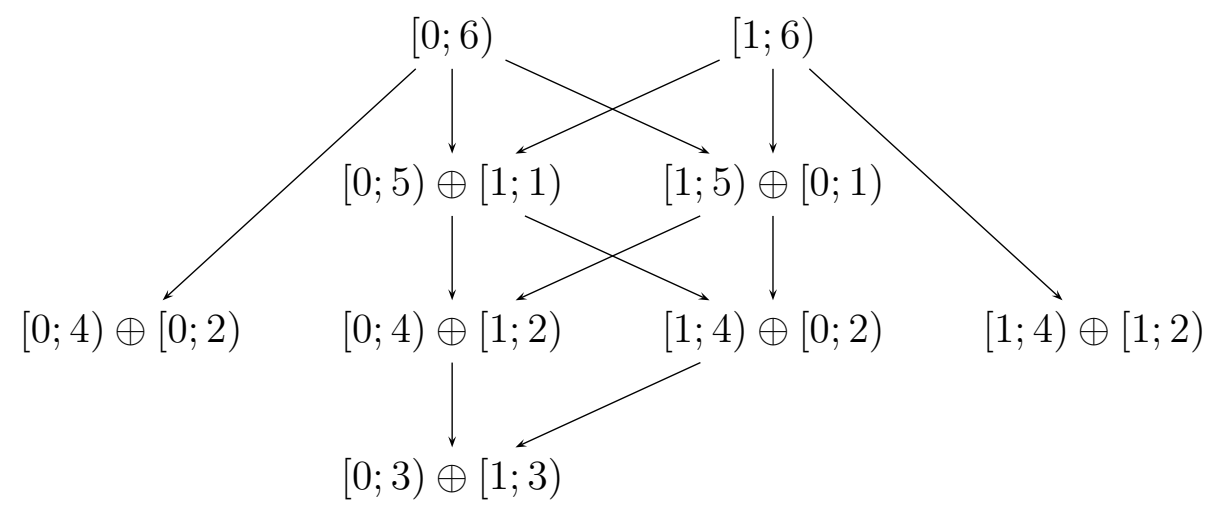




\section{Proof of Theorem 3.1}

For $\boldsymbol{\lambda} \geq \boldsymbol{\mu}$ in $\boldsymbol{\Lambda}^{\leq 2}(\mathbf{d})$, define a nonnegative integer $c_{\boldsymbol{\lambda}, \boldsymbol{\mu}}$ by the following rule. If $\boldsymbol{\lambda}=\boldsymbol{\mu}$, let $c_{\boldsymbol{\lambda}, \boldsymbol{\mu}}=1$. If $\boldsymbol{\lambda}>\boldsymbol{\mu}$, then $\boldsymbol{\mu} \in \boldsymbol{\Lambda}^{\leq 2}(\mathbf{d})_{<\boldsymbol{\lambda}}^{i_{1}, i_{2}}$ for unique $\left(i_{1}, i_{2}\right)$, and the interval $[\boldsymbol{\mu}, \boldsymbol{\lambda}]$ is contained in $\boldsymbol{\Lambda}^{\leq 2}(\mathbf{d})_{\leq \boldsymbol{\lambda}}^{i_{1}, i_{2}}$. Define $s_{1}, s_{2}$, and $s$ as above, and let $k(\boldsymbol{\mu})=\min \left\{s_{1}, s_{2}\right\}$. Say $\boldsymbol{\mu}$ is special (relative to $\boldsymbol{\lambda}$ ) if there is no element $\boldsymbol{\nu}$ of $[\boldsymbol{\mu}, \boldsymbol{\lambda}]$ with $k(\boldsymbol{\nu})=k(\boldsymbol{\mu})$ except $\boldsymbol{\mu}$ itself. If $\boldsymbol{\mu}$ is special, let $c_{\boldsymbol{\lambda}, \boldsymbol{\mu}}=\left(\begin{array}{c}s \\ k(\boldsymbol{\mu})\end{array}\right)-\left(\begin{array}{c}s \\ k(\boldsymbol{\mu})-1\end{array}\right)$. Otherwise, let $c_{\boldsymbol{\lambda}, \boldsymbol{\mu}}=0$. We can now prove a stronger version of Theorem 3.1:

Theorem 7.1. If $\boldsymbol{\lambda} \geq \boldsymbol{\mu}$ in $\boldsymbol{\Lambda}^{\leq 2}(\mathbf{d})$, then $\tilde{K}_{\boldsymbol{\lambda}, \boldsymbol{\mu}}(t)=1, a_{\boldsymbol{\lambda}, \boldsymbol{\mu}, j}=0$ if $j \neq 0$, and $a_{\boldsymbol{\lambda}, \boldsymbol{\mu}, 0}=c_{\boldsymbol{\lambda}, \boldsymbol{\mu}}$.

Proof. By inspection of the diagrams in the previous section, we see that if $\boldsymbol{\mu}$ is special, then $\epsilon(\boldsymbol{\mu})-\epsilon(\boldsymbol{\lambda})=2 k(\boldsymbol{\mu})$. Moreover, for all positive $k^{\prime} \leq k(\boldsymbol{\mu})$, there is a unique special $\boldsymbol{\nu} \in[\boldsymbol{\mu}, \boldsymbol{\lambda}]$ such that $k(\boldsymbol{\nu})=k^{\prime}$. So Corollary 5.7 can be rephrased:

$$
g_{\boldsymbol{\lambda}, \boldsymbol{\mu}}(t)=\sum_{\boldsymbol{\lambda} \geq \boldsymbol{\nu} \geq \boldsymbol{\mu}} c_{\boldsymbol{\lambda}, \boldsymbol{\nu}} t^{(\epsilon(\boldsymbol{\nu})-\epsilon(\boldsymbol{\lambda})) / 2} .
$$

Now we apply Lemma 4.2 (bearing in mind Remark 4.3) with $b_{\boldsymbol{\lambda}, \boldsymbol{\mu}, j}=$ $\delta_{j, 0} c_{\boldsymbol{\lambda}, \boldsymbol{\mu}}$ and $L_{\boldsymbol{\lambda}, \boldsymbol{\mu}}(t)=1$. The result follows.

\section{REFERENCES}

[1] N. Chriss and V. Ginzburg, Representation Theory and Complex Geometry, Birkhauser Boston, 1997.

[2] J. J. Graham and G. I. Lehrer, Diagram algebras, Hecke algebras and decomposition numbers at roots of unity. Preprint.

[3] A. Henderson, Fourier transform, parabolic induction, and nilpotent orbits, Transform. Groups, 6 (2001), pp. 353-370.

[4] D. Kazhdan and G. Lusztig, Proof of the Deligne-Langlands conjecture for Hecke algebras, Invent. Math., 87 (1987), pp. 153-215.

[5] B. Leclerc, J.-Y. Thibon, and E. Vasserot, Zelevinsky's involution at roots of unity, J. Reine Angew. Math., 513 (1999), pp. 33-51.

[6] G. Lusztig, Green polynomials and singularities of unipotent classes, Adv. Math., 42 (1981), pp. 169-178.

[7] — Canonical bases arising from quantized enveloping algebras, J. Amer. Math. Soc., 3 (1990), pp. 447-498.

[8] I. G. Macdonald, Symmetric Functions and Hall Polynomials, Oxford Univ. Press, second ed., 1995.

School of Mathematics and Statistics, University of Sydney, NSW 2006, AUSTRALIA

E-mail address: anthonyh@maths.usyd.edu.au 